\title{
Comment on: External Validation of the Simplified Preoperative Assessment for Low-Grade Mucinous Adenocarcinoma of the Appendix
}

\author{
Sean P. Dineen, $\mathrm{MD}^{1}$, Richard E. Royal, $\mathrm{MD}^{2}$, Paul F. Mansfield, $\mathrm{MD}^{2}$, and Keith F. Fournier, $\mathrm{MD}^{2}$ \\ ${ }^{1}$ GI Oncology, Moffitt Cancer Center, Tampa; ${ }^{2}$ Surgical Oncology, MD Anderson Cancer Center, University of Texas, \\ Houston
}

\section{TO THE EDITORS:}

We read with much interest the article by Milovanov et al. that was recently published in Annals of Surgical Oncology. ${ }^{1}$ The authors reported an external validation of a simple scoring system we had previously published for determining the likelihood of a complete cytoreduction in patients with low-grade mucinous adenocarcinoma of the appendix (the SPAAT score). ${ }^{2}$ In this external validation, there was a notable difference in the positive predictive value (PPV) of the SPAAT score compared with our original publication (50\% vs. $95 \%$ ). In addition, the authors noted a negative predictive value (NPV) of $90 \%$, indicating that a SPAAT score $\geq 3$ predicted failure to achieve a complete cytoreduction. The authors conclude that the SPAAT score has limited clinical utility due to low sensitivity and PPV.

The analysis by Milovanov et al. prompts several thoughts. First, in our original article, 28 patients in the validation cohort had a SPAAT score $\geq 3$, while in this external validation only six patients had a SPAAT score $\geq 3$, limiting the strength of their assessment. Second, despite their small numbers, a SPAAT score $\geq 3$ retained a high NPV. Thus, patients with a preoperative assessment demonstrating a SPAAT score $\geq 3$ can be effectively counseled on the high likelihood of an incomplete cytoreduction, and patient expectations adjusted accordingly. In our opinion, this is of critical clinical importance, and the publication by Milovanov et al. validates the use of this score for such a purpose. Finally, of the six patients with a SPAAT score $<3$ who underwent incomplete cytoreduction, five had small bowel disease precluding resection. It is somewhat surprising that none of these patients had foreshortening of the mesentery on preoperative computed tomography imaging. In developing our scoring system, we identified patients from 2008 to 2009, while, in contrast, Milovanov et al. used patients dating as far back as 1998. The improvements in imaging quality during that time frame are not inconsequential and could explain the identification of this finding.

In summary, we agree that further revision of scoring systems (ours and others) will be useful in improving care for patients with peritoneal disease; however, we would argue that the SPAAT score continues to have important clinical utility, particularly in identifying patients who are unlikely to derive significant survival benefit from attempted cytoreductive surgery/hyperthermic intraperitoneal chemotherapy (CRS/HIPEC).

\section{REFERENCES}

1. Milovanov V, Sardi A, Aydin N, et al. External validation of the simplified preoperative assessment for low-grade mucinous adenocarcinoma of the appendix. Ann Surg Oncol. 2017; 24(7):1783-86.

2. Dineen SP, Royal RE, Hughes MS, et al. A simplified preoperative assessment predicts complete cytoreduction and outcomes in patients with low-grade mucinous adenocarcinoma of the appendix. Ann Surg Oncol. 2015; 22(11):3640-6.

(C) Society of Surgical Oncology 2017

First Received: 27 September 2017;

Published Online: 9 November 2017

S. P. Dineen, MD

e-mail: sean.dineen@moffitt.org 